\title{
Haptic Rendering of Topological Constraints to Users Manipulating Serial Virtual Linkages
}

\author{
Daniela Constantinescu and Septimiu E. Salcudean \\ Electrical \& Computer Engineering Department \\ University of British Columbia \\ Vancouver, B.C., Canada \\ danielac@ece.ubc.ca,tims@ece.ubc.ca
}

\author{
Elizabeth A. Croft \\ Department of Mechanical Engineering \\ University of British Columbia \\ Vancouver, B.C., Canada \\ ecroft@mech.ubc.ca
}

\begin{abstract}
This paper presents an approach for haptic rendering of topological constraints to users operating serial virtual linkages. In the proposed approach, a haptic device controller is designed to penalize users' departure from the configuration manifold of the virtual linkage. This manifold is locally approximated through the range space of the Jacobian of the virtual linkage computed at the user's hand. Simulations and controlled experiments performed using a planar haptic interaction system demonstrate that the proposed approach successfully constrains the users' motion as required by the topology of the virtual linkage that they manipulate.

Index Terms-topological constraints, serial linkage, physically-based simulation, haptic rendering.
\end{abstract}

\section{INTRODUCTION}

Haptic interaction with physically-motivated virtual environments provides an understanding of the virtual world that can not be obtained using other human-computer communication paradigms. At the same time, haptics bring about specific computational difficulties. A main challenge is the need to compute the dynamics and the interactions of the virtual objects at guaranteed speeds of the order of hundreds of Hz. This computational requirement poses difficulties for the rendering of haptic interaction between a user and a virtual environment through a linkage and only a very few examples [1], [2], [3], [4] have been reported. Nevertheless, potential applications such as virtual CAD prototyping, medical simulators for orthopedics, or animation require users to be provided with convincing force feedback while they manipulate virtual linkages.

The main factors influencing the realism of the haptic manipulation of linkages are: (i) the physical accuracy of the linkage simulation; (ii) the users' perception of the linkage dynamics and of its contacts; and, (iii) the users' perception of the topological constraints. Existing research has developed application-specific [1] and generalpurpose [2], [3], [4], [5] haptic rendering techniques that address the first two factors. In [1], [2], and [4] the main concern is the efficient simulation of the linkage dynamics. Physically-based models for linkage contact and collision are developed in [3] and [5]. However, users are allowed to operate the linkage only from links with redundant degrees of freedom. In addition, with the exception of [5], only point interaction with virtual linkages is enabled.
The present research is the first to propose and demonstrate an approach for imposing topological constraints on users during their rigid body manipulation of serial linkages from any user-selected link. In the proposed approach, users' departure from the configuration manifold of the virtual linkage is penalized through device control. This control uses the singular directions of the linkage Jacobian computed at the user's hand to enforce the virtual topology.

The paper starts by briefly overviewing the control architecture in Section II. The virtual linkage simulation is introduced in Section III. The users' perception of the inertia of the virtual linkage is discussed in Section IV. The controller proposed for representing the topological constraints to the haptic device is developed in Section V. Simulations illustrating that this controller can be used to tightly constrain users to the configuration manifold of the virtual linkage that they manipulate (hereafter also called virtual tool - VT) are presented in Section VI. The efficient implementation of the proposed approach is detailed in Section VII. Haptic rendering of virtual topology is experimentally demonstrated in Section VIII. Conclusions are presented in Section IX.

\section{Control Architecture}

In this work, the control architecture proposed in [6] is used for haptic rendering of user interaction within rigid multibody virtual environments (VEs). This architecture comprises an impedance-type [7] device controller and a four channel teleoperation [8] haptic controller. The device controller changes the impedance of the haptic device to match the impedance of the VT. The haptic controller applies the linkage contacts to the user's hand. A one degree of freedom (DOF) mechanical equivalent of this control architecture (excluding the communication delay) is depicted in Fig. 1. In this figure, $Z_{d}$ and $Z_{v t}$ are the impedance of the device and of the VT, respectively, $S$ scales the two force channels (from the device to the VE and vice-versa), and $f_{h}, f_{e n v}$, and $f_{p c}$ are the hand, the $\mathrm{VE}$, and the coordination force ${ }^{1}$, respectively. If $Z_{d}=Z_{v t}$

\footnotetext{
${ }^{1}$ The coordination force is applied by the position coordination channels of the haptic controller. These channels are shown in Fig. 1 as a springdamper connection between the device and the VT.
} 
and forces are not scaled $(S=1)$ the haptic controller is transparent. Users perceive the linkage inertia and the motion constraints imposed by the linkage topology through $Z_{v t}$. They perceive the motion constraints imposed by other virtual objects through $f_{e n v}$.

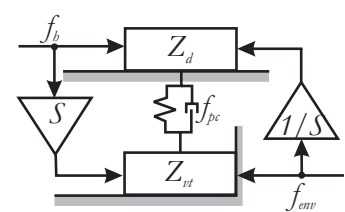

Fig. 1. 1 DOF mechanical equivalent (excluding the communication delay) of the four channel teleoperation controller for the case of onedimensional haptic interaction.

\section{LinKAGE Simulation}

Physically-based linkage simulation can be performed either in Cartesian space or in configuration space. In Cartesian space, the dynamics of each link are derived independently and the linkage topology is imposed through algebraic constraint equations. Hence, a computationally expensive differential algebraic system of equations must be integrated for which topological constraint satisfaction may be problematic [9]. In configuration space, the linkage topology is embedded in the coordinate representation. Only a reduced set of coordinates must be integrated and the satisfaction of the topological constraints is guaranteed. Therefore, linkages are simulated in configuration space in this work.

Since the coordinate representation embeds the topological constraints, only the contact and the user-applied forces must be included in the dynamic equations. Hence, the configuration space dynamics of a linkage (i.e., VT) with $d$ DOFs and $c$ contacts are given by:

$\mathbf{D}(\mathbf{q}) \ddot{\mathbf{q}}+\mathbf{B}(\mathbf{q}, \dot{\mathbf{q}})+\mathbf{G}(\mathbf{q})=\sum_{i=1}^{c} \mathbf{J}_{i}^{T}(\mathbf{q}) \mathbf{F}_{i}+\mathbf{J}_{h}^{T}(\mathbf{q}) \mathbf{F}_{h}$

In (1), notation is used as follows: $\mathbf{D}(\mathbf{q})_{d \times d}$ is the configuration space mass matrix of the VT; $\mathbf{B}(\mathbf{q}, \dot{\mathbf{q}})_{d \times 1}$ represent Coriolis and centripetal effects; $\mathbf{G}(\mathbf{q})_{d \times 1}$ are the gravitational terms; $\mathbf{J}_{i}(\mathbf{q})_{6 \times d}$ is the linkage Jacobian computed at the $i$-th contact; $\mathbf{F}_{i_{6 \times 1}}=\left(\begin{array}{ll}\mathbf{f}_{i}^{T} & \mathbf{0}^{T}\end{array}\right)^{T}$ is the contact wrench (i.e., the force $\mathbf{f}_{i_{3 \times 1}}$ and torque $\boldsymbol{\tau}_{i_{3 \times 1}}=$ $\left.\mathbf{0}_{3 \times 1}\right)$ at the $i$-th contact; $\mathbf{J}_{h}(\mathbf{q})_{6 \times d}$ is the linkage Jacobian computed at the user's hand (hand Jacobian); $\mathbf{F}_{h_{6 \times 1}}$ is the wrench applied by the user; and $\mathbf{q}_{d \times 1}, \dot{\mathbf{q}}_{d \times 1}$, and $\ddot{\mathbf{q}}_{d \times 1}$ are the configuration space position, velocity, and acceleration of the virtual linkage, respectively. Note that (1) represents the dynamics of a passive VT. Topological constraints are imposed on users manipulating active virtual linkages via adding joint torques to (1).

In (1), the contact wrenches $\mathbf{F}_{i}$ model the multiple contact interactions between the VT and other objects in the VE. They are computed as described in [5]. Once computed, (1) can be directly solved for the VT configuration space acceleration:

$$
\ddot{\mathbf{q}}=\mathbf{D}^{-1}\left(\sum_{i=1}^{c} \mathbf{J}_{i}^{T} \mathbf{F}_{i}+\mathbf{J}_{h}^{T} \mathbf{F}_{h}-\mathbf{B}-\mathbf{G}\right) .
$$

In (2), the instantaneous state dependence of all terms on the right hand side of the equation is implied. The VT simulation is advanced via integrating the configuration space acceleration of the VT using a forward integrator with time step equal to the time step of the control loop.

While the dynamics of the virtual linkage are simulated in configuration space, users interact with it in Cartesian space. Therefore, the linkage inertia, the topological constraints, and the contact forces must be applied to the user's hand in Cartesian space. The representation of the linkage inertia to the user is discussed in the following section, while the approach proposed for representing the topological constraints to the device is presented in Section V.

\section{LINKAGE INERTIA AT THE USER-SELECTED POINT}

Both the inertia and the topological constraints are embedded in the operational space inertia of the linkage computed at the user's hand, $\boldsymbol{\Lambda}_{h_{6 \times 6}}$, [10]:

$$
\boldsymbol{\Lambda}_{h}=\left(\mathbf{J}_{h} \mathbf{D}^{-1} \mathbf{J}_{h}^{T}\right)^{-1} \text {. }
$$

Therefore, $\boldsymbol{\Lambda}_{h}$ is incorporated in this work in the desired impedance of the VT, $Z_{v t}$. Specifically, the device controller changes the dynamics of the device to match the desired VT dynamics [6]:

$$
\Lambda_{h} \ddot{\mathbf{x}}_{h}+\mathbf{b}_{d} \dot{\mathbf{x}}_{h}+\mathbf{k}_{d} \mathbf{x}_{h}=\mathbf{F}_{h}+\mathbf{F}_{e n v}+\mathbf{F}_{p c} .
$$

In (4), $\boldsymbol{\Lambda}_{h}, \mathbf{b}_{d_{6 \times 6}}$, and $\mathbf{k}_{d_{6 \times 6}}$ are the desired (i.e., simulated) inertia, damping, and stiffness of the VT, respectively, $\mathbf{F}_{p c_{6 \times 1}}$ is the coordination wrench between the device and the VT (due to the generalized spring-damper shown in Fig. 1), and $\ddot{\mathbf{x}}_{h_{6 \times 1}}, \dot{\mathbf{x}}_{h_{6 \times 1}}$, and $\mathbf{x}_{h_{6 \times 1}}$ are the desired body acceleration, velocity, and position of the device at the user-selected operational point, respectively.

Equation (4) is equivalent to:

$$
\ddot{\mathbf{x}}_{h}=\boldsymbol{\Lambda}_{h}^{-1}\left(\mathbf{F}_{h}+\mathbf{F}_{e n v}+\mathbf{F}_{p c}-\mathbf{b}_{d} \dot{\mathbf{x}}_{h}-\mathbf{k}_{d} \mathbf{x}_{h}\right),
$$

where $\Lambda_{h}^{-1}$ is given by:

$$
\Lambda_{h}^{-1}=\mathbf{J}_{h} \mathbf{D}^{-1} \mathbf{J}_{h}^{T} .
$$

Since $\mathbf{D}$ is invertible, $\boldsymbol{\Lambda}_{h}^{-1}$ can be computed regardless of the rank of $\mathbf{J}_{h} \cdot \Lambda_{h}^{-1}$ embeds the directions of topological constraint at the user's hand in its singular directions. To illustrate how the constraints imposed on the user's motion by the VT topology are represented in the rank-defficiency of $\boldsymbol{\Lambda}_{h}^{-1}$, consider the example manipulation of the planar linkage depicted in Fig. 2. In this example, the user holds the linkage from the centre of mass (COM) of the middle link in the position shown in Fig. 2. The held link has 
insufficient DOFs to allow arbitrary position and orientation at the user-selected point. Hence, the linkage topology instantaneously constrains the user's motion according to:

$$
\dot{\mathbf{x}}_{h}=\mathbf{J}_{h} \dot{\mathbf{q}},
$$

where the hand Jacobian is:

$$
\mathbf{J}_{h}=\left[\begin{array}{ccc}
-l_{1} s_{\left(\theta_{1}\right)}-l_{c_{2}} s_{\left(\theta_{1}+\theta_{2}\right)} & -l_{c 2} s_{\left(\theta_{1}+\theta_{2}\right)} & 0 \\
l_{1} c_{\left(\theta_{1}\right)}+l_{c_{2}} c_{\left(\theta_{1}+\theta_{2}\right)} & l_{c 2} c_{\left(\theta_{1}+\theta_{2}\right)} & 0 \\
1 & 1 & 0
\end{array}\right] .
$$

Equation (8) shows that $\operatorname{rank}\left(\mathbf{J}_{h}\right)=2$. From (6), it follows that $\operatorname{rank}\left(\boldsymbol{\Lambda}_{h}^{-1}\right)=2$. Hence, $\boldsymbol{\Lambda}_{h}^{-1}$ drops rank when the VT restricts the user's motion.

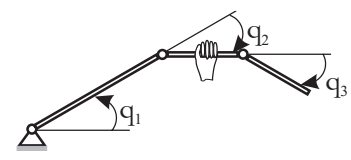

Fig. 2. Example rigid body manipulation of a planar linkage. The user holds the middle link and the linkage topology prevents them from achieving arbitrary position and orientation.

Since $\boldsymbol{\Lambda}_{h}^{-1}$ can be computed regardless of the rank of $\mathbf{J}_{h}$, (5) is used in this work for the impedance control of the device. This equation computes the desired body acceleration at the user's hand. Given the device dynamics:

$$
\mathbf{M}_{d} \ddot{\mathbf{x}}_{h}+\mathbf{C}_{d} \dot{\mathbf{x}}_{h}=\mathbf{F}_{h}+\mathbf{u},
$$

where $\mathbf{M}_{d}$ and $\mathbf{C}_{d}$ are the device inertia and Christoffel matrices, and $\mathbf{u}$ is the control signal [6], the impedance control law for the interface is obtained as:

$$
\begin{aligned}
\mathbf{u}= & \left(\mathbf{M}_{d} \boldsymbol{\Lambda}_{h}^{-1}-\mathbf{I}\right) \mathbf{F}_{h}+\mathbf{M}_{d} \boldsymbol{\Lambda}_{h}^{-1}\left(\mathbf{F}_{e n v}+\mathbf{F}_{p c}\right) \\
& +\left(\mathbf{C}_{d}-\mathbf{M}_{d} \boldsymbol{\Lambda}_{h}^{-1} \mathbf{b}_{d}\right) \dot{\mathbf{x}}_{h}-\mathbf{M}_{d} \boldsymbol{\Lambda}_{h}^{-1} \mathbf{k}_{d} \mathbf{x}_{h} .(10)
\end{aligned}
$$

Equation (10) enforces the VT topology at the acceleration level. Due to numerical drift and limited device stiffness, (5) is not sufficient for realistic haptic rendering of the topological constraints. A technique that enables users to convincingly perceive these constraints is proposed in the following section.

\section{LINKAGE TOPOLOGY AT THE USER-SELECTED POINT}

An analysis of the VT dynamics suggests how the control in (10) can be augmented to enable users to convincingly perceive linkage topology. At the user-selected point, $\boldsymbol{\Lambda}_{h}^{-1}$ maps $\mathbf{F}_{\text {env }}$ to the VT body acceleration at the user's hand:

$$
\ddot{\mathbf{x}}_{h}=\boldsymbol{\Lambda}_{h}^{-1} \mathbf{F}_{e n v} .
$$

In (11), $\ddot{\mathbf{x}}_{h}=\left(\begin{array}{ll}\dot{\mathbf{v}}_{h}^{T} & \dot{\boldsymbol{\omega}}^{T}\end{array}\right)^{T}$ belongs to the space of body accelerations at the user's hand, $A$; i.e., $\dot{\mathbf{v}}_{h_{3 \times 3}}$ and $\dot{\boldsymbol{\omega}}_{3 \times 3}$ are the linear and the angular acceleration of the user's hand, respectively. When $\boldsymbol{\Lambda}_{h}^{-1}$ is full rank, $\mathbf{F}_{e n v}$ produces body acceleration of the user's hand along all directions of $A$ and the VT behaves as an inertia along all directions of
$A$. On the control side, (10) applies this inertia to users by controlling their acceleration to the value given in (11).

When $\boldsymbol{\Lambda}_{h}^{-1}$ is rank-deficient, $\ddot{\mathbf{x}}_{h}$ lies in a subspace of $A$. Then, environment wrenches exist that lie in the null space of $\boldsymbol{\Lambda}_{h}^{-1}, \mathcal{N}\left(\boldsymbol{\Lambda}_{h}^{-1}\right)$. These wrenches are opposed by the structural stiffness of the VT and have no effect on the body acceleration at the user's hand. Hence, at the user-selected point, the linkage behaves as an infinite stiffness along these directions and behaves as an inertia along directions orthogonal to them. On the control side, the control in (10) is unsuitable for rendering the infinite structural stiffness of the VT along the directions in $\mathcal{N}\left(\Lambda_{h}^{-1}\right)$.

It can be shown that the null spaces of $\Lambda_{h}^{-1}$ and $\mathbf{J}_{h}^{T}$ are equal, i.e., that the two mappings have the same singular directions. Physically, this can be understood by considering the action of $\mathbf{J}_{h}^{T}$. Specifically, $\mathbf{J}_{h}^{T}$ maps the space of wrenches $F$ to the space of configuration torques $T$ :

$$
\tau=\mathbf{J}_{h}^{T} \mathbf{F}_{h},
$$

i.e., to the space of torques that result in body acceleration at the user's hand. In other words, $\mathbf{J}_{h}^{T}$ filters out from the configuration space dynamics of the VT the components of the hand wrench that do not influence this acceleration. Therefore, $\mathcal{N}\left(\mathbf{J}_{h}^{T}\right)=\mathcal{N}\left(\boldsymbol{\Lambda}_{h}^{-1}\right) . \mathcal{N}\left(\boldsymbol{\Lambda}_{h}^{-1}\right)$ is called the space of topological constraint and computed using the singular value decomposition (SVD) of $\mathbf{J}_{h}$ in this work. Moreover, its orthogonal complement (the range space of $\mathbf{J}_{h}, \mathcal{R}\left(\mathbf{J}_{h}\right)$ ) is called the space of topological freedom of motion.

Realistic perception of the topological constraints is enabled through augmenting the control provided by (10) with penalties applied along the directions of topological constraint, as shown in Fig. 3. In this figure, $v t$ is the user's position and orientation on the VT (i.e., in the VE), while $h$ is their real position and orientation (i.e., on the device). For simplicity, only a one dimensional $\mathcal{N}\left(\mathbf{J}_{h}^{T}\right)$ is depicted.

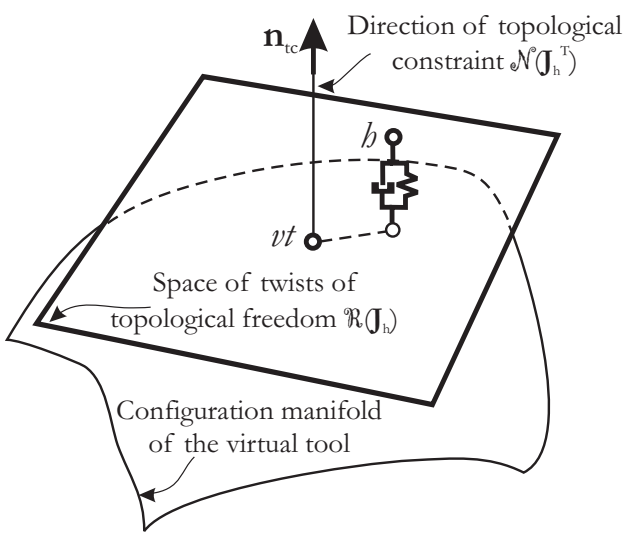

Fig. 3. Penalty wrench constraining users to the configuration manifold of the virtual linkage that they manipulate.

The directions of topological constraint $\mathbf{n}_{t c}$ are provided 
by $\mathcal{N}\left(\mathbf{J}_{h}^{T}\right)$. The constraint position and velocity are provided by the body position $\mathbf{x}_{h_{v t}}$ and velocity $\dot{\mathbf{x}}_{h_{v t}}$ of the user-selected point on the VT. Then, given the stiffness $k_{t c}$ and damping $b_{t c}$ of the topological constraints, the control law in (10) is augmented with the control signal:

$$
\begin{aligned}
\mathbf{u}_{a d d}= & \mathbf{M}_{d} \sum_{i=1}^{t}\left(k_{t c} \mathbf{n}_{t c, i}^{T}\left(\mathbf{x}_{h_{v t}}-\mathbf{x}_{h}\right)\right. \\
& \left.+b_{t c} \mathbf{n}_{t c, i}^{T}\left(\dot{\mathbf{x}}_{h_{v t}}-\dot{\mathbf{x}}_{h}\right)\right) \mathbf{n}_{t c, i},
\end{aligned}
$$

where $t$ is the number of topological constraints. Note that $t=6-\operatorname{rank}\left(\mathbf{J}_{h}\right)$ during spatial rigid body (6 DOFs) manipulation, and $t=3-\operatorname{rank}\left(\mathbf{J}_{h}\right)$ during planar rigid body (3 DOFs) manipulation.

Hence, the proposed control is:

$$
\begin{aligned}
\mathbf{u}= & \left(\mathbf{M}_{d} \boldsymbol{\Lambda}_{h}^{-1}-\mathbf{I}\right) \mathbf{F}_{h}+\mathbf{M}_{d} \boldsymbol{\Lambda}_{h}^{-1}\left(\mathbf{F}_{e n v}+\mathbf{F}_{p c}\right) \\
& +\left(\mathbf{C}_{d}-\mathbf{M}_{d} \boldsymbol{\Lambda}_{h}^{-1} \mathbf{b}_{d}\right) \dot{\mathbf{x}}_{h}-\mathbf{M}_{d} \boldsymbol{\Lambda}_{h}^{-1} \mathbf{k}_{d} \mathbf{x}_{h} \\
& +\mathbf{M}_{d} \sum_{i=1}^{t}\left(k_{t c} \mathbf{n}_{t c, i}^{T}\left(\mathbf{x}_{h_{v t}}-\mathbf{x}_{h}\right)\right. \\
& \left.+b_{t c} \mathbf{n}_{t c, i}^{T}\left(\dot{\mathbf{x}}_{h_{v t}}-\dot{\mathbf{x}}_{h}\right)\right) \mathbf{n}_{t c, i}
\end{aligned}
$$

The ability of this control to impose on users the topology of the VT is validated through simulations in the following section and through controlled experiments in Section VIII.

\section{Simulations}

In this section, a simulated manipulation of a threelink planar virtual linkage is used to compare the performance of the control in (14) to the performance of the control in (10) developed in [6]. In the simulation, users manipulate the VT depicted in Fig. 4 by applying a constant force $f_{h}=0.4(\mathrm{~N})$ along the $x$ direction at the COM of the distal link. The linkage parameters are given in Table I. Initially, the VT is at rest in the position $\mathbf{q}_{0}=\left(-\frac{\pi}{4}(\mathrm{rad}) \quad \frac{\pi}{6}(\mathrm{rad})-\frac{\pi}{6}(\mathrm{rad})\right)^{T}$. The haptic device is also at rest, but its position differs from that of the user-selected operational point by $5(\mathrm{~mm})$ along the $x$ direction and by $3(\mathrm{~mm})$ along the $y$ direction (see Fig. 4).

TABLE I

PARAMETERS OF THE THREE-LINK PLANAR VIRTUAL LINKAGE OPERATED BY THE USER IN THE SIMULATIONS.

\begin{tabular}{|l|l|l|}
\hline Link length $(\mathbf{m})$ & Link mass $(\mathrm{kg})$ & Link inertia $\left(\mathrm{kg} \cdot \mathbf{m}^{2}\right)$ \\
\hline$l_{1}=0.042(\mathrm{~m})$ & $m_{1}=3(\mathrm{~kg})$ & $I_{1}=0.015\left(\mathrm{~kg} \cdot \mathrm{m}^{2}\right)$ \\
$l_{2}=0.042(\mathrm{~m})$ & $m_{2}=3(\mathrm{~kg})$ & $I_{2}=0.015\left(\mathrm{~kg} \cdot \mathrm{m}^{2}\right)$ \\
$l_{3}=0.030(\mathrm{~m})$ & $m_{3}=1(\mathrm{~kg})$ & $I_{3}=0.005\left(\mathrm{~kg} \cdot \mathrm{m}^{2}\right)$ \\
\hline
\end{tabular}

The Simulink ${ }^{T M}$ diagram of the manipulation is shown in Fig. 5. In the diagram, the VE is represented by the configuration space linkage dynamics, while the haptic device controlled according to (10) is represented by the operational space linkage dynamics. The additional control signal used to enforce linkage topology on users $\mathbf{u}_{\text {add }}$ is

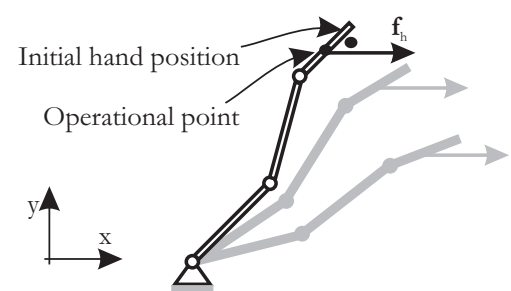

Fig. 4. Simulated manipulation of a virtual linkage. Initial VT position is shown in black. VT positions during manipulation are shown in grey.

explicitly represented. Moreover, $\mathbf{M}_{d}$ is considered constant throughout the workspace and equal to unity. The coordination between the device and the VT has stiffness:

$$
\mathbf{K}_{p c}=(100(\mathrm{~N} / \mathrm{m}) \quad 100(\mathrm{~N} / \mathrm{m}) \quad 0.5(\mathrm{~N} \cdot \mathrm{m} / \mathrm{rad}))^{T},
$$

and damping:

$\mathbf{B}_{p c}=\left(\begin{array}{lll}70(\mathrm{~N} /(\mathrm{m} / \mathrm{s})) & 70(\mathrm{~N} \cdot \mathrm{m} /(\mathrm{rad} / \mathrm{s})) & 0.375(\mathrm{~N} /(\mathrm{m} / \mathrm{s})))^{T} .\end{array}\right.$

These values are chosen to match the values implemented in the haptic controller used in the experiments [6]. The stiffness and damping of the topological constraints are $k_{t c}=10(\mathrm{~N} /(\mathrm{m} \cdot \mathrm{kg}))$ and $b_{t c}=7((\mathrm{~N} \cdot \mathrm{s}) /(\mathrm{m} \cdot \mathrm{kg}))$, respectively.

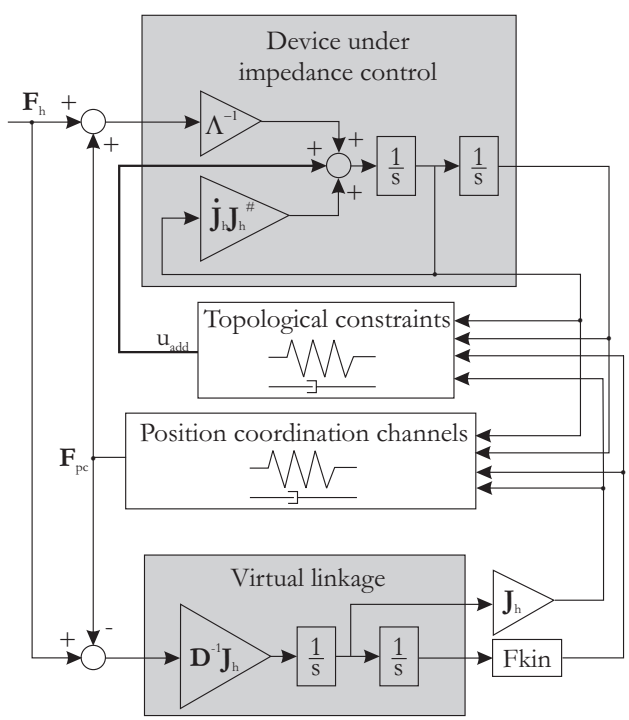

Fig. 5. Simulink diagram of the haptic manipulation of a virtual linkage.

The simulation results are plotted in Fig. 6. The user's hand trajectories on the device and in the VE are shown in Fig. 6(a) for the case when topology is imposed at the acceleration level. They are shown in Fig. 6(b) for the case when user's departure from the constraint manifold of the VT is penalized according to (13). Since the user holds a link with 3 DOFs, the linkage topology constrains the user only intermittenly, when the user moves through a singularity. Note that the device drifts from the VT when linkage constraints are imposed at the acceleration 
level. In contrast, the drift is substantially reduced through penalizing user's violation of the virtual topology.

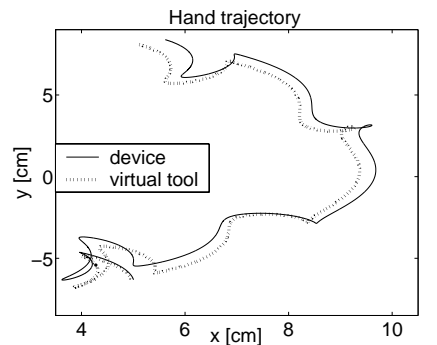

(a) Topology imposed at the acceleration level.

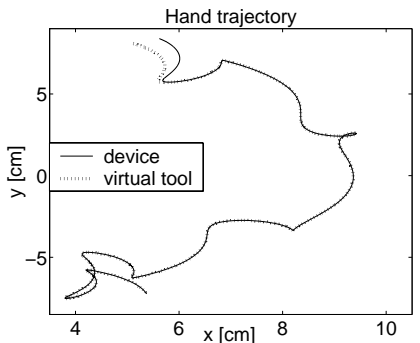

(b) Topology imposed through penalties.
Fig. 6. Simulated manipulation of a planar three-link VT from the COM of the distal link.

The control in (14) requires the computation of the directions of topological constraints $\mathbf{n}_{t c}$. This computation is based on the SVD of $\mathbf{J}_{h}$, which may be numerically expensive for the speed requirements of the haptic control loop. The following section discusses suitable approximations of these directions that can be implemented in a local model of interaction with guaranteed real time performance.

\section{IMPLEMENTATION}

To avoid the computation of the singular directions of $\mathbf{J}_{h}$, the force control loop is decoupled from the VE simulation through a local model of interaction (LMI) [11] (see Fig. 7). The LMI is a reduced simulation that runs at the frequency of the force control loop. It approximates the interaction between the VT and the VE through the interaction between the VT and nearby objects. The quality of the approximation is maintained by updating the LMI at each step of the VE simulation.

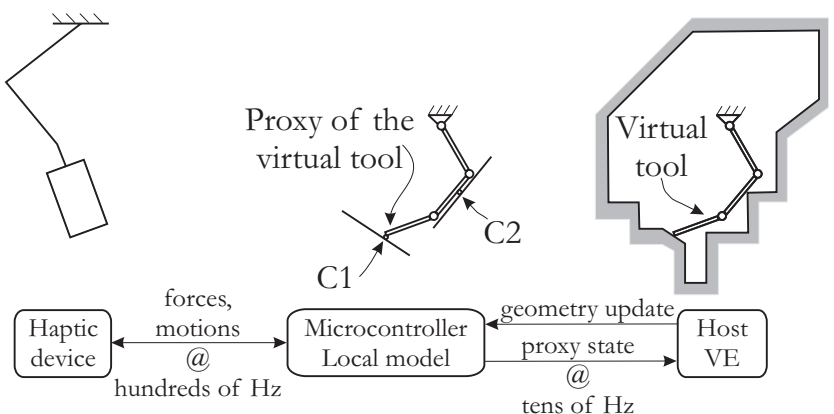

Fig. 7. Decoupling of the force control loop from the VE simulation through a LMI.

The computational load of the LMI is much reduced, since the collision detection bottleneck is eliminated and only the VT dynamics are simulated. During haptic manipulation of linkages, the numerical efficiency of the LMI is further increased by approximating the configuration space inertia of the linkage and the gravitational terms with their values in the $\mathrm{VE}$ at the moment of the update, and by ignoring Coriolis and centripetal effects (similar to work in [2] and [4]), i.e., by simulating:

$$
\widehat{\mathbf{D}} \ddot{\mathbf{q}}+\widehat{\mathbf{G}}=\sum_{i=1}^{c} \mathbf{J}_{i}^{T} \mathbf{F}_{i}+\mathbf{J}_{h}^{T} \mathbf{F}_{h} .
$$

In (17), $\widehat{\mathbf{D}}$ and $\widehat{\mathbf{G}}$ denote the values of $\mathbf{D}$ and $\mathbf{G}$, respectively, computed by the VE and sent to the LMI at the update. Furthermore, the directions of topological constraint are approximated through their values in the VE at the update. In other words, the SVD of $\mathbf{J}_{h}$ is computed by the VE simulation:

$$
\widehat{\mathbf{J}}_{h}=\widehat{\mathbf{U}} \widehat{\mathbf{\Sigma}} \widehat{\mathbf{V}}^{T}
$$

and the columns of $\widehat{\mathbf{U}}$ are used in the LMI to estimate the directions of topological constraint. Lastly, $\Lambda_{h}^{-1}$ is approximated locally by:

$$
\widehat{\boldsymbol{\Lambda}}_{h}^{-1}=\widehat{\mathbf{J}}_{h} \widehat{\mathbf{D}}_{v t}^{-1} \widehat{\mathbf{J}}_{h} .
$$

Equations (17), (18), and (19) provide significant computational savings in the LMI and allow the VT dynamics to be simulated at the frequency of the haptic controller. Operation of virtual linkages using these approximations is demonstrated experimentally in the following section.

\section{EXPERIMENTS}

The users' perception of the VT topology was investigated through a controlled experiment performed using the planar haptic interaction system available in the Robotics and Control Laboratory at the University of British Columbia [11]. In the experiment, the user operates the three-link planar virtual linkage shown in Fig. 4 from the COM of its distal link (dimensions and inertial properties are given in Table I). Trajectories obtained by enforcing the topological constraints through (14) (i.e., through penalizing user's departure from the configuration manifold) are contrasted to trajectories obtained by enforcing the virtual topology through (10) (i.e., through controlling to zero user's acceleration along the directions orthogonal to the configuration manifold of the VT).

A constant wrench $\mathbf{F}_{h}=\left(\begin{array}{lll}0.4(\mathrm{~N}) & 0(\mathrm{~N}) & 0(\mathrm{~N} \cdot \mathrm{m})\end{array}\right)^{T}$ represents the user ${ }^{2}$ in the experiment. The linkage is initially at rest, in the configuration space position shown

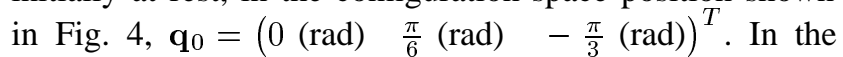
LMI, the linkage dynamics are approximated by:

$$
\widehat{\mathbf{D}} \ddot{\mathbf{q}}=\mathbf{J}_{h}^{T} \mathbf{F}_{h},
$$

i.e., the VT moves freely under the controlled hand wrench. The topological stiffness and damping are

${ }^{2}$ For the impedance type haptic interface employed in these experiments, a constant wrench represents a worst case approximation for stability. 
$k_{t c}=200(\mathrm{~N} /(\mathrm{m} \cdot \mathrm{kg}))$ and $b_{t c}=50((\mathrm{~N} \cdot \mathrm{s}) /(\mathrm{m} \cdot \mathrm{kg}))$, respectively.

The experimental device trajectory ('HD') and the simulated hand trajectory ('VE') are plotted in Fig. 8. Fig. 8(a) shows that the experimental trajectory follows the simulated trajectory with a steady state error due to the limited stiffness of the penalties applied to users. Hence, (14) effectively imposes the topological constraints on users operating the linkage through singularities. On the other hand, Fig. 8(b) shows that users drift from the configuration manifold of the VT (i.e., from the simulated trajectory) until they reach the mechanical boundary of the workspace of the haptic device. The sudden changes occuring in the experimental trajectory after approximately $0.8 \mathrm{~s}$ are due to the hard constraints imposed on users by the workspace limits of the haptic interface. Hence, the impedance controller alone cannot enforce the virtual topology.

\section{CONCLUSION}

This paper has presented an approach for haptically rendering the linkage topology to users manipulating serial virtual linkages. In this approach, the topological constraints have been imposed on users through penalizing users' violation of the configuration manifold of the virtual linkage. A local approximation of this manifold has been computed using the singular directions of the linkage Jacobian at the user's hand. The proposed approach allows the stiffness and damping of the topological constraints to be chosen independently of the stiffness and damping of the virtual contacts in the haptic simulation. The ability to impose the virtual topology on users operating serial linkages has been illustrated through simulations and experiments performed using a planar haptic interaction system.

Future work will investigate the extension of the proposed approach to linkages with arbitrary topologies, including multiple closed loops.

\section{REFERENCES}

[1] A. Nahvi, D. Nelson, J. Hollerbach, and D. Johnson, "Haptic Manipulation of Virtual Mechanisms from Mechanical CAD Designs," in Proceedings of the 1998 IEEE International Conference on Robotics and Automation, Leueven, Belgium, May 1998, pp. 375-380.

[2] D. Ruspini and O. Khatib, "Dynamic Models for Haptic Rendering Systems," in Advances in Robot Kinematics: ARK98, Strobl/Salzburg, Austria, 1998, pp. 523-532.

[3] _ - "Collision/Contact Models for Dynamic Simulation and Haptic Interaction," in Proceedings of the 9th International Symposium on Robotics Research, Snowbird, UT, 1999, pp. 185-195.

[4] - "Haptic Display for Human Interaction with Virtual Dynamic Environments," Journal of Robotic Systems, vol. 18, no. 2, pp. 769783, 2001.

[5] D. Constantinescu, S. Salcudean, and E. Croft, "Haptic Rendering of Rigid Contacts using Impulsive and Penalty Forces," Accepted by the IEEE Transactions on Robotics, August 2004.

[6] M. Sirouspour, S. DiMaio, S. Salcudean, P. Abolmaesumi, and C. Jones, "Haptic Interface Control - Design Issues and Experiments with a Planar Device," in Proceedings of the 2000 IEEE International Conference on Robotics and Automation, San Francisco, Ca, 2000, pp. 789-794.
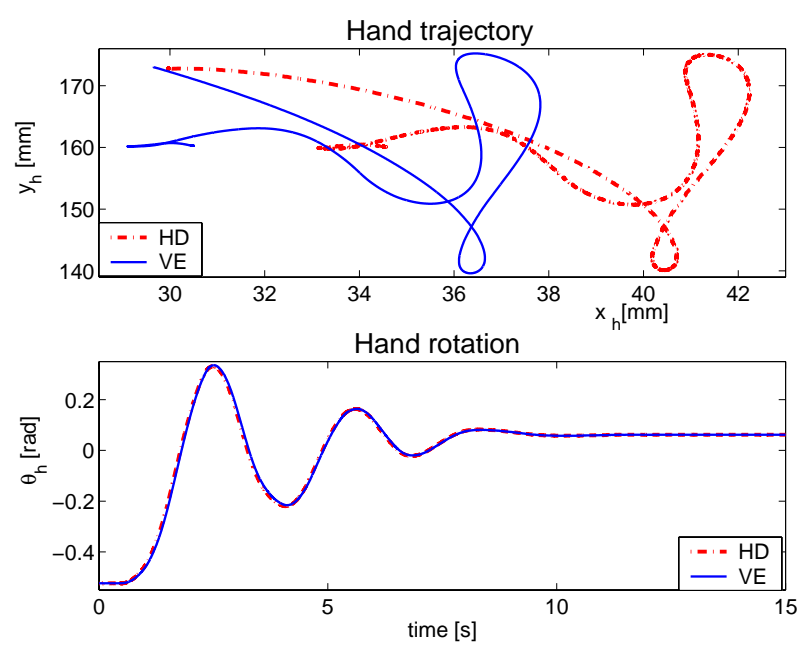

(a) Topology enforced through penalties.
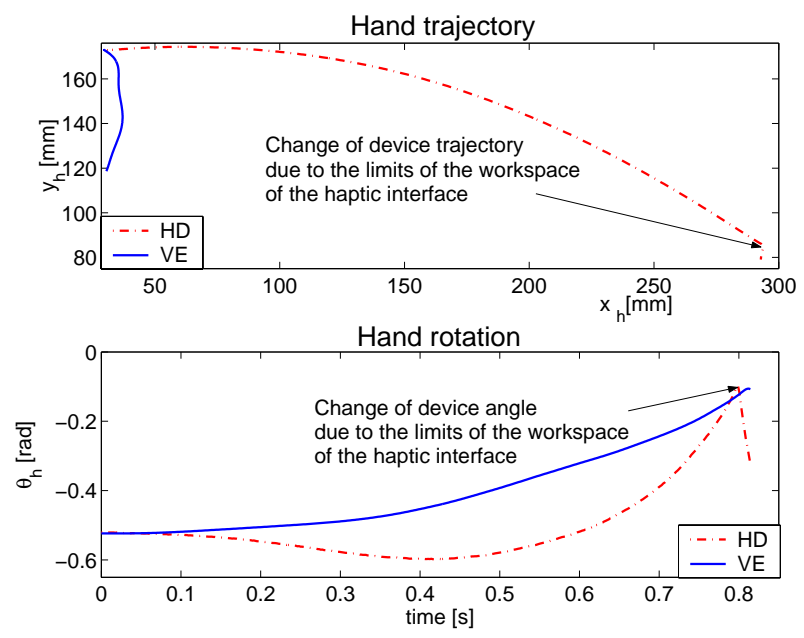

(b) Topology enforced at acceleration level.

Fig. 8. Haptic manipulation from the distal link of the virtual linkage shown in Figure 4.

[7] N. Hogan, "Impedance Control: An Approach to Manipulation. Theory, Implementaion, Application," Transactions of the ASME, Journal of Dynamic Systems, Measurement, and Control, vol. 107, pp. 1-24, March 1985.

[8] D. Lawrence, "Stability and Transparency in Bilateral Teleoperation," IEEE Transactions on Robotics and Automation, vol. 9, no. 5, pp. 624-637, 1993.

[9] D. Ruspini, K. Koralov, and O. Khatib, "The Haptic Display of Complex Graphical Environments," in Proceeding of the SIGGRAPH 97, Los Angeles, CA, 1997, pp. 345-352.

[10] O. Khatib, "A Unified Approach for Motion and Force Control of Robot Manipulators: The Operational Space Formulation," IEEE Transactions on Robotics and Automation, vol. 3, no. 1, pp. 43-53, 1987.

[11] D. Constantinescu, S. Salcudean, and E. Croft, "Haptic Feedback using Local Models of Interaction," in 11th International Symposium on Haptic Interfaces for Virtual Environment and Teleoperator Systems, Los Angeles, Ca, 2003, pp. 416-421. 